\title{
A Human-Animal-Robot Cooperative System for Anti-Personal Mine Detection
}

\author{
Thrishantha Nanayakkara, Tharindu Dissanayake, Prasanna Mahipala, \\ and K. A. Gayan Sanjaya \\ University of Moratuwa \\ Sri Lanka
}

\begin{abstract}
This chapter reports the latest results of an attempt made in Sri Lanka to develop a humananimal-robot integrated system to detect anti-personal landmines. In this study, a mongoose was trained to sniff for landmines. It was attached to a semi-autonomous legged mobile robot. The robot could sense the direction of movement of the mongoose. The robot used this direction information along with commands sent by a remote human operator to modify its own semi-autonomous behaviors. The human operator gave overall commands as to which direction the robot should move. The robot took care of detailed tasks such as obstacle avoidance, monitoring the environment immediately in front of it, and guiding the mongoose. The mobile robot was a laboratory made fully embedded platform with simple sensors such as bumper switches and a sonar sensor. Experiments were carried out in typical environments where anti-personal landmines are buried in Sri Lanka.

\section{Introduction}

One of the most significant challenges faced by any post war human re-settlement program is to cover a vast unstructured land of minefields as fast as possible with a guarantee of safety for civilians. For most of the developing countries, landmine removal is a prerequisite to economic development. Apparently, demining is an operation accompanied with a lot of risk to human deminers. Therefore, manual deminers move slowly. Analysis of the movements of the human deminers shows that the mobility part can be easily and safely performed by robotic systems. Then the challenge is reduced to sensing the landmines.

\section{is 1.1 Landmine and Explosive Detection Technologies}

D A survey has been done to categorize different technologies available for landmine detection

principle observed and reported, class-2 is for those with technology concept and/or application formulated, class-3 for those with analytical and experimental critical function and/or characteristic proof of concept is given, class-4 is for those with technology

Source: Humanitarian Demining: Innovative Solutions and the Challenges of Technology, Book edited by: Maki K. Habib, ISBN 978-3-902613-11-0, pp. 392, February 2008, I-Tech Education and Publishing, Vienna, Austria
\end{abstract}


component and/or basic technology sub-system validation in laboratory environment has been done, class- 5 is for those with technology component and/or basic sub-system validation in relevant environment, Class-6 is for those with technology system/subsystem model or prototype demonstration in a relevant environment has been done, Class-7 is for those with technology system prototype demonstration in an operational environment, Class- 8 is for those with actual technology system completed and qualified through test and demonstration, and Class-9 is for those with technology system "accredited" through successful mission operations.

\subsubsection{Metal Detectors (Electromagnetic Induction Devices)}

A time varying current in a transmitting coil induces an "eddy" current in nearby metallic objects. The magnetic field created by this induced current induces a Voltage in a receiving coil of the metal detector. This signal is filtered and amplified to generate an acoustic signal that works as the alarm (Collins L., et al, 2002). The major limitations of this technology are: It gives a lot of false alarms for shell fragments, bullet cases, and other metal debris usually found in mine fields, it is difficult to tune in laterite rich soil or conductive soil (red soil, sea beaches etc.), it is difficult to detect mines with more than 1 feet soil deposits over it, and there can be false alarms due to electromagnetic interference.

\subsubsection{Ground Penetrating Radar (GPR)}

GPR works by emitting a microwave signal into the soil. If an object such as a landmine that has a different dielectric property than the surrounding soil is present, the sensor can use the reflected signal to differentiate the object and the soil (Sun Y. \& Li J., 2003; Savelyev T.G., et al, 2007). Therefore, GPR does not look for the small metal parts in the landmine like in metal detectors. The major limitations are: It is an expensive technology. Therefore it is very seldom used in the affected regions in the world; Microwaves attenuate in conductive and wet soils. It gives false alarms for roots, rocks, water pockets etc., there is a need to compromise between the resolution and penetration. Resolution is high at elevated frequencies, and penetration is high at low frequencies. In (Sun Y. \& Li J., 2003), experiments have been done using a GPR detector mounted in front of a field vehicle so that the GPR detector is directed forward. When the vehicle moves forward, the data is collected in time domain. The frequency analysis of these data has been done. 2-D images have been obtained by plotting the real part of the Fourier transformed data on the $X$-axis and the frequency spectrum on the Y-axis. The corresponding image gives different patterns for different mines buried at different depths. This could be considered an effective method to detect suspicious items by visual inspection. Yet, how this method will perform when the sensor array vibrates due to the jolt of the vehicle when it moves in a rough terrain is questionable.

\subsubsection{Multisensor Systems Using GPR and Metal Detectors}

1. Hand Held Standoff Mine Detection System (HSTAMIDS or acronym AN/PSS-14) (Xu X., et al, 2002): This US army project has developed a multi-sensor system that contains a GPR and a Metal Detector that is used by a sensor fusion algorithm for feature extraction.

2. MINEHOUND (Doheny R.C., et al, 2005) is claimed to be simple and effective compared to other GPR based technologies. The project is sponsored by the UK Department for International Development and developed by ERA Technology. 
3. ALIS (Advanced Landmine Detection System) (Daniels D.J., et al, 2005) similar to HSTAMIDS, this uses GPR and Pulse Induced Metal Detectors. The limitations are: it is very expensive for normal use, and need a large platform to carry it, that may cause problems in a tropical minefield.

There has been considerable amount of effort given to improve the sensor fusion algorithms (Sato M., et al, 2005; Ferrari S. \& Vaghi A., 2006). In (Sato M., et al, 2005), a sensor fusion technique based on Bayesian networks is proposed. The method is tested on a system of GPR, electromagnetic induction, and infra-red sensors. The data have been processed as batches. Therefore, there is no guarantee that this method can be deployed in an unstructured environment with no prior information to detect landmines on line.

\subsubsection{Trace Explosive Detection Systems}

Samples of the environment have to be obtained and the detection of explosives is done based on a chemical reaction or a mass-spectroscopy measurement (Perrin S., et al, 2004; George V., et al, 1999; Cumming C., et al, 2001). For instance, one technology is to use Amplifying Fluorescent Polymers (AFPs) (George V., et al, 1999). In the absence of explosive agents, the polymer fluoresces when exposed to light. With the presence of nitro-aromatic compounds, the fluorescence decreases. The limitations are: Slow to respond, expensive, requires samples of the environment and therefore not efficient to detect on the move.

\subsubsection{Biosensors}

These use a Quartz crystal microbalance (QCM) together with antibody $(\mathrm{Ab})$ and Antigens (Ag). A sample collector sucks in the vapor. If there are substrates of TNT/DNT, RDX, PETN and tetryl, the oscillating frequency of the QCM changes due to the migration of the micro-organisms (Fisher M.E. \& Sikes J., 2003; Crabbe S., 2005; BIOSENS Consortium, 2004). This change in the frequency is detected to give an alarm. Limitations: Slow response, can have a drift due to population changes, very expensive.

\subsubsection{Magnetic Quadrupole Resonance}

A method is proposed to observe the quadrupole resonance signals of explosives that are often interfered by background radio signals (Crabbe S., et al, 2003). For instance in TNT, there are 18 resonant frequencies 12 of which are between $700-900 \mathrm{kHz}$ that is susceptible to interference from AM radio. Since the resonant signal is weak, filtering and observation is necessary. This method proposes to use the noise as the state and the signal as the measurement noise of the Kalman filter. If the interference from AM radio is handled with improved signal processing methods, this method can be useful as a remote sensing technique. However, the technology is not yet mature.

\subsubsection{Seismo-Acoustic Methods}

Seismic waves are generated from one end and from the other end, a non-contact (acoustic) transducer and a contact (seismic) transducer picks up the signal (Tan Y., et al, 2005; Xiang N. \& Sabatier J., 2004; Scott W.R., et al, 2001]. If there are hollow objects like landmines, it will be reflected in the received signal because of its difference with the mechanical properties of the surrounding soil. Attempts have been taken to identify the vibration 
signature of a mine. However, ageing and changes in the mechanical properties of the mine may lead to false alarms.

In essence, given the scale of the need to have a technology to detect landmines and explosives, the current state of the art is very expensive, computationally cumbersome, and gives high false alarm rates. Therefore, there is an open need to have a simple, cost effective, and powerful technique that can be applied in most practical cases. We believe the answer lies in a system that combines the strengths of animals to detect chemical agents, semiautonomous robots that can navigate in a cluttered environment to guide the animal, and a human's ability to control a robot and to analyze the visual feedback of an animal's behavior.

\subsection{The Proposed Method to Use a Rodent}

The most common animal used for explosive detection all around the world is the dog. In addition to dogs there are situations where rats and bees have been used for this kind of purposes. Although these animals are used today, there are certain drawbacks and limitations of them. Dogs' sniffing capability is powerful in downward direction and not that strong upwards like in searching a vehicle. In addition they are not much suitable for work in crowded places. Rats have been used to sniff and detect Tuberculosis Bacteria in human sputum samples. Yet, due to limited stamina, they can not cover large areas and work continuously. The primary sensing system in our approach is a trained mongoose. It's said to have the third most sniffing ability among animals, only being less than that of elephant and pig. Furthermore unlike dogs, it has a powerful sniffing ability in upward direction. The rodent is attached to a semi-autonomous field robot through an elastic cord with an angle sensor at the robot's end. The angle sensor will provide information about the rodent's wondering behaviors.

\section{Training a Mongoose to Detect Landmines}

\subsection{Animal-robot Interaction}

Experiments have been done to observe how chicken behaves when a robot elicits different behaviors in the same cage (Bohlen M., 1999). It has been found that the chicken reacts to the relative velocities and accelerations of the robot, the sounds it generate, and how close it comes to them. Sometimes, animals attach value to colors. Experiments done on male Sticklebacks have shown that any odd shaped thing with a red bottom will make them think that it is a threat to them, because stickleback males show a red belly during courtship season to differentiate them from females (De Schutter G., et al, 2001). Experimentsconducted on miniature robots working in societies of cockroaches show that once the insects accept the robot to be part of their society, the robot can influence some of the collective behaviors of the animals such as foraging under a shade, moving as a group etc. (Caprari G., et al, 2005). An algorithm to accelerate reward based training of rats is proposed in (Ishii H., et al, 2005), where a rat learns to press levers of a robot to obtain food and water. It is found that the training process should be phased out in order to accelerate training. In this case, the first phase was to remove the anxiety of the rat to face the robot, the next phase was to reward the robot for coming closer the robot, the last phase was to motivate the robot to press the appropriate levers of the robot to get food and water. Based on the work done so far on understanding how different animals react to the presence of 
robots, and how an animal can be trained to interact with a robot, we expect to investigate the features of the robot's appearance and the behaviors in order to make the best use of the animal's natural sensory system. A major novelty of the proposed study is that the robot does not duplicate any hardware needed to sense the environment wherever the animal can do it better. Hence the robot becomes simpler and cheaper. Furthermore, the robot learns from the animal how to move in a cluttered environment while restricting the animal's movements to a given area of interest.

In the area of forcing an animal to behave in a given way, some work has been done on invasive techniques such as invoking behaviors through artificial stimulation of the animal's nervous system. A Bio-robotic system has been explained where a cockroach is electrically stimulated to turn left and right to keep it on a black strip (Holzer R. \& Shimoyama I, 1997). A similar attempt to guide a rat along a desired path is described in (Talwar S.K., et al, 2002), where electrodes were planted in the rat's somatosensory cortical whisker representations to give sensory cues, and the Medial forebrain bundles to give the rewards. The rat has been guided along a given path using a wireless data communication system between a backpack mounted on the rat and a remote supervisor. However, it is our belief that invasive techniques should be avoided wherever possible in order to make the best use out of the animal's natural sensory system.

Monitoring of animal's behaviors from a remote place or directly monitoring the activities of the brain is of paramount importance in the proposed study. The advances in PET technology have been very useful in brain imaging of animals (Vaska P., et al, 2004; Ziegler S.I., 2005). This could open up future opportunities to trace the activity of the olfactory system of the animals and match it against a template for a particular type of explosive. Yet, this remains a remote possibility given the current state of the art. Some work has been done to model animal behavior using 3-D video images (Ramanan D., et al, 2006; Girard M., 1987). A possible extension is to capture a particular behavior that the animal might elicit in response to hazardous objects. Yet, in the proposed study, a human in a remote control room will do the behavior tracking of the animal. This method will render itself to be robust with minimum errors.

\subsection{Swarm Robots}

Swarm robotics is a related area of research where a complex collective behavior is emerged in a group of relatively simple robots through inter-robot and robot-environment interactions (Goldberg D. \& Mataric M., 1997; Hayes A, 2002; Johns C. \& Mataric M., 2003; Krieger M. \& Billeter J.B., 2003). A given robot is generally very simple and inexpensive, capable of eliciting a limited array of primitive behaviors, and equipped with a limited number of sensors and actuators. Given a task to be accomplished such as walking over a gap that none of the individual robots can not accomplish, the simple robots may share their diverse sensor information, actively support each other by joining hands, and coordinate movements to achieve the common goal. This concept can be useful in a task to find a hazardous object in a cluttered environment where one robot can not carry all the required sensors. Yet in that case, there is nothing that improves the behavior of an individual robot. The focus is to emerge a collective behavior based on the rules that apply to each robot to interact with other robots and the environment, and not to improve individual robotic behaviors through peer interaction. To the best of our knowledge there has been no work done to study a scenario where each robot is directly influenced by a real animal as a peer 
who can sniff for the target objects and at the same time support the robot to learn how to navigate in a cluttered environment.

\subsection{Characterizing the Environment}

Experiments have been done in Sri Lanka where a robot navigates in a vegetated tropical minefield using a single sonar sensor (Nanayakkara T., et al, 2006). The sonar scans the environment to derive statistics of the distances between the robot and the trees. In one scan, it collects 10 readings of the distances to the trees. Based on the variance and the mean of the distances to the trees obtained in one scan, the robot classifies the environment into one of a given number of known environments. An image based 3-D map building approach for forest environments has been proposed in (Forsman P. \& Halme A., 1998). Yet, how far this can be applied in a minefield is questionable, because the robot has to recognize the environment in the first trial. The sonar and laser range finder based map building and robot localization has been studied extensively in the recent history especially in indoor environments (Thrun S., et al, 1998; Burgard W., et al, 1998; Ayache N. \& Faugeras O.D., 1989; Bozma O. \& Kuc R., 1991; Chong K.S. \& Kleeman L., 1996; Dudek G., et al, 1996; ). In most cases, a sonar sensor is used to alert the laser range finder to look for finer details. In a forest or a densely wooded environment or in a highly congested area, the sonar will always give alerts. Therefore, these methods may not be as good as having an animal to signal the robot to change the path to avoid an obstacle.

\subsection{Advantages of Using a Mongoose}

○ Mongooses have a highly developed sense of smell;

- Mongooses are easy to tame and train;

- Mongooses are small, cheap and easy to maintain and transport;

- Once taught, the animals love performing repetitive tasks;

- It has found that the mongoose can exceed the sensitivity of dogs appear to be well founded;

○ Mongooses are widespread and easily adapt to all environments.

\subsection{Biological Facts about the Mongoose}

The mongoose belongs to Herpestidae family. They can be found in Asia, Africa, the Caribbean and southern Europe. There are more than thirty species, ranging between one and four feet in length.

Mongoosesare mostly carnivores, feeding on insects, crabs, earthworms, lizards, snakes, rodents and other small creatures.

They will also consume eggs, carrion and sometimes fruit. Some species, such as the Indian mongoose, are popularly known for their ability tofight and kill venomous snakes such as cobras. They are able to do this because of their speed, agility and cunning properties. 


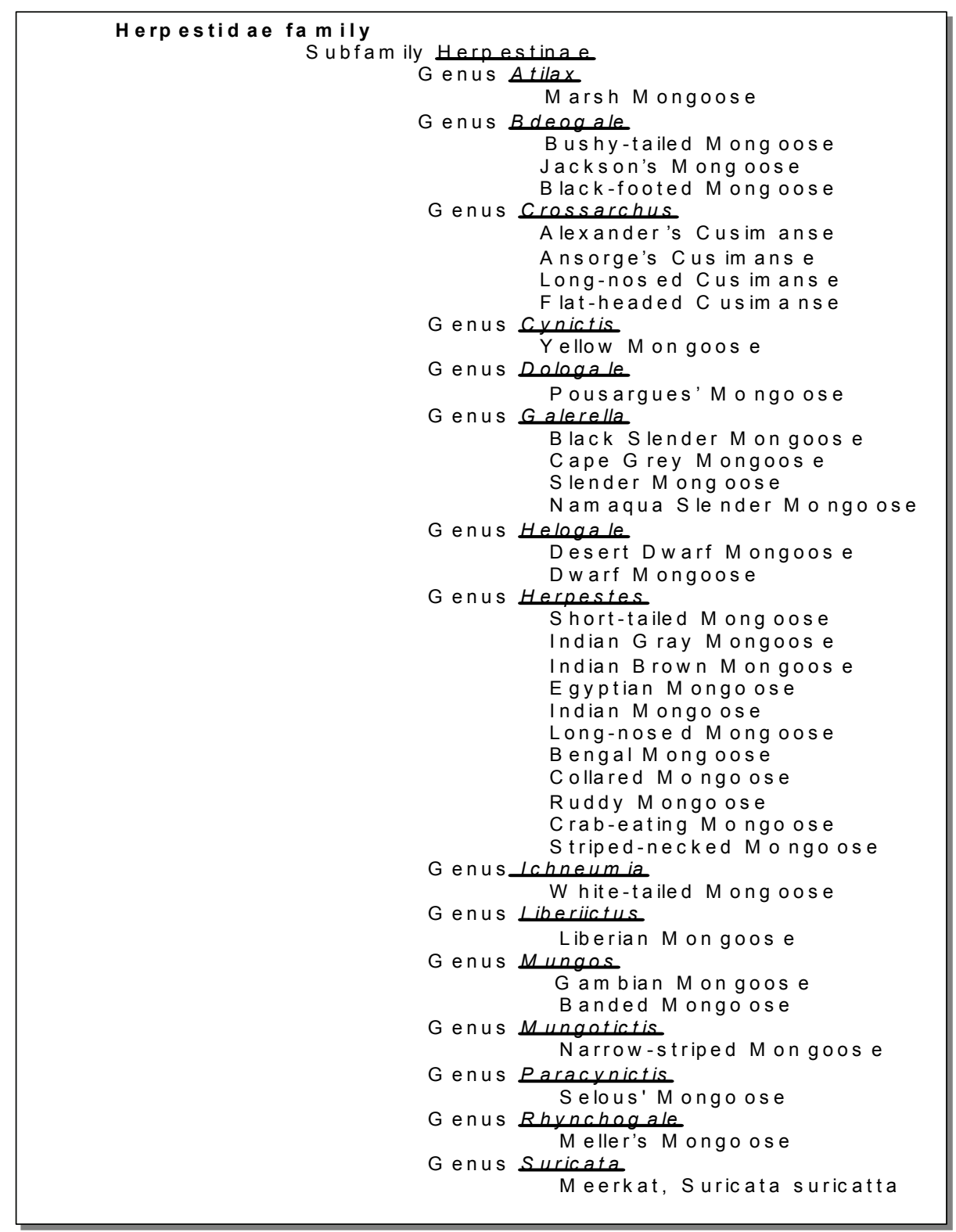

Among those species what we find here in Sri Lanka mostly is Dwarf Mongoose. 


\subsection{Reward Based Learning of Mongooses to Detect Explosives}

The plasticity of an animal's brain can be effectively used to develop a motivation to carry out tasks that it did not use to enjoy. Basically the animal is repeatedly given a reward whenever it carries out a desired task. This type of learning is known as reward based learning or reinforcement based learning (Schultz W., et al, 1997; Dayan P. \& Balleine B., 2002; Montague P. \& Berns G., 2002; Hollerman J.R., et al, 1998; German P.W. \& Fields H.L., 2007). Experimental results on how the activity of the Dopamine neurons in the brain is related to reward based learning suggests that the brain tries to give priority to actions that maximize the total expected rewards given by equation (1), where $V(t)$ is the total expected reward, $E(\bullet)$ is the expectation function, $r(t)$ is the reward at time $t$, and $\gamma$ is the discounting rate.

$$
V(t)=E\left(r(t)+\gamma r(t+1)+\gamma^{2} r(t+2)+\cdots\right)
$$

However, mathematical models developed so far in the machine learning literature suggest that the learning speed largely depends on the shape of the reward function (Sutton R.S. \& Barto A.G., 1998; Watkins C.J.C.H, 1989; Mataric M.J., 1994; Mataric M.J., 2000; Ng A.Y., et al, 1999). In machine learning, a control policy $\pi$ is updated using a critic that estimates $V(t)$ given a situation $s$ and a policy $\pi$. The policy is defined as $\pi: s \rightarrow a$, where $s$ is the situation and $a$ is the action. Therefore, it is clear that the speed and accuracy of the process of improving the control policy depends on the accuracy of the critic that estimates $V(t)$ given in equation (1).

Work done on motor memory consolidation using human subjects suggest that learning in distinctive episodes leads to the formation of robust modular internal representations that can be combined to form complex skills (Shadmehr R. \& Holcomb H.H., 1997; BrushersKrug T., et al, 1996; Thoroughman K. \& Shadmehr R., 2000). A salient feature of these modules of internal representations is that they are less prone to interference from others. In other words, the parameter changes in one module have less influence on the performance of another module. Therefore, we expect the proposed learning method to simplify the cost landscape in the learning parameter space, thus accelerating convergence of parameters towards a global optimum.

Based on the above scientific background, we started our experiments with two mongooses. They were of the dwarf mongoose type. The first step was to train the wild mongooses that are sensitive to even the slightest disturbance in the environment to interact with humans and be impartial to sudden disturbances in the environment. In the initial phases fish and meat were tested as a medium for rewards. Yet, we soon realized that the traces of raw fish and meat emanated a strong smell that overshadowed the smell of explosives in small quantities. Among other foods that did not have a strong smell such as cake, apple, banana, cheese, and milk, the mongoose demonstrated a great affinity to cheese.

\subsection{Experimental Results of Training Mongooses to Detect Explosives}

The mongooses were trained by bringing a small amount of $\mathrm{C} 4$ explosives wound to one end of a stick while observing the behavior of the animal. If the mongoose came closer to the stick, we gave a "beep" sound with a slice of cheese as the reward. The sound helped us to 
get the attention of the mongoose. As stated earlier we had two mongooses. One was small and the other one was larger in size. Both mongooses did not show much improvement during the first ten days. But the larger one made a sudden improvement during the next few days. It started to learn to detect explosives. When we moved the stick with explosives nearer, it tried to pull the stick and search for cheese. Gradually we decreased the amount of explosives attached. At the beginning we had to insert the stick into the cage for the mongoose to detect it. Gradually we kept it away as much as possible and at last we were able to make it detect keeping the stick at the outside edge of the cage. After some time, it became eager to look for explosives upon hearing the "beep" sound. Furthermore, as we moved the stick along the cage it followed the path. Following figure $\mathbf{1}$ gives an idea of how the mongoose improved during our experiment. We did the test twenty times a day.

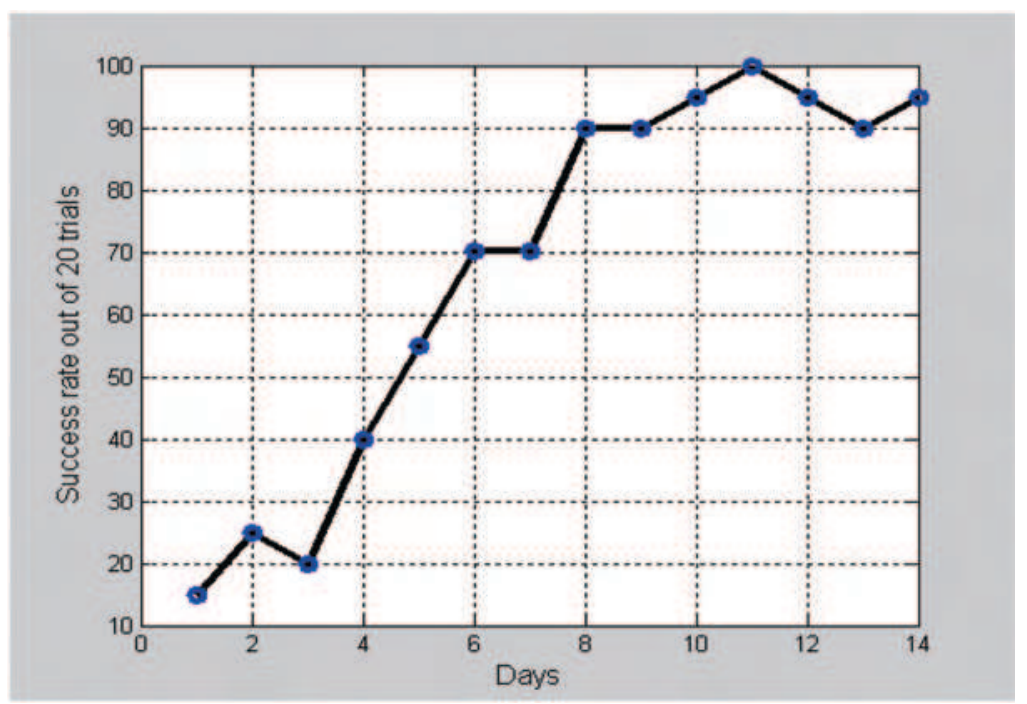

Fig. 1. The daily improvement of the mongoose's ability to associate explosives to an anticipated reward

Two weeks after this improvement, we extended our experiments in several alternative paths. We wanted to give the mongoose the idea that it will receive the reward if and only if it detected explosives. Therefore, we used several sticks. One was without explosives. Another had an edge covered by a cloth, but without explosives inside. We used another stick with a cloth attached and gave it the smell of a perfume. The fourth stick had explosives attached at the end. Different sticks were presented to the mongoose in blocks of 40 trials. Over time, the number of times the stick with explosives was presented was brought down to test the accuracy of the training. After two and half weeks time it was able to successfully distinguish between several similar types of sticks, and find the correct stick containing explosives. 


\begin{tabular}{|l|l|l|l|}
\hline Day & $\begin{array}{l}\text { No. of times } \\
\text { explosive stick } \\
\text { wased out of } \\
40 \text { trials (X) }\end{array}$ & $\begin{array}{l}\text { Successful Trials } \\
\text { (out of } \mathrm{x} \text { ) }\end{array}$ & $\begin{array}{l}\text { Percentage of Success } \\
(\%)\end{array}$ \\
\hline 1 & 26 & 4 & 15.38 \\
\hline 2 & 28 & 3 & 10.71 \\
\hline 3 & 26 & 6 & 23.08 \\
\hline 4 & 21 & 8 & 38.10 \\
\hline 5 & 18 & 7 & 38.89 \\
\hline 6 & 17 & 8 & 47.06 \\
\hline 7 & 17 & 9 & 52.94 \\
\hline 8 & 15 & 7 & 46.67 \\
\hline 9 & 15 & 8 & 53.33 \\
\hline 10 & 16 & 10 & 62.50 \\
\hline 11 & 13 & 9 & 69.23 \\
\hline 12 & 14 & 11 & 78.57 \\
\hline 13 & 10 & 9 & 90 \\
\hline 14 & 9 & 9 & 100 \\
\hline 15 & 10 & 8 & 80 \\
\hline 16 & 8 & 7 & 87.50 \\
\hline 17 & 9 & 9 & 100 \\
\hline
\end{tabular}

Table 1: The improvement of the ability of mongoose to detect a stick with explosives among similar sticks without explosives

Having succeeded with the above training phase, we moved on to remove all visual cues available to the mongoose by covering the cage with a black screen. The sticks with or without explosives were brought closer to the cage from behind these screens. In order to attract the attention of the mongoose to sniff for explosives, we tapped on the cover before moving the sticks. Gradually it learnt to move towards the cover by sensing the smell. At present the mongoose has become able to distinguish among several smells.

\section{Mongoose - Robot Coupled System}

The primary role of the robot is to guide the mongoose along a desired path. After a number of experiments with different robotic platforms, we designed a robot that looks and walks like an Iguana frequently seen in tropical forests in Sri Lanka. Figure 2 shows the laboratory made robot that weighs $4 \mathrm{~kg}$. The trained mongoose is attached to the robot with an elastic cord. The cord is attached to the robot through an angle sensor that allows the robot to sense the direction of drag of the mongoose. The legged robot consists of two independent units, each resembling the shape of an Iguana. The two units are kept together using two rods hinged at the front and the rear end of each unit. Two units are driven by two geared DC 
motors that can be controlled independently. Therefore, turning involves one unit moving faster than the other or one unit reversing while the other unit moves forward.
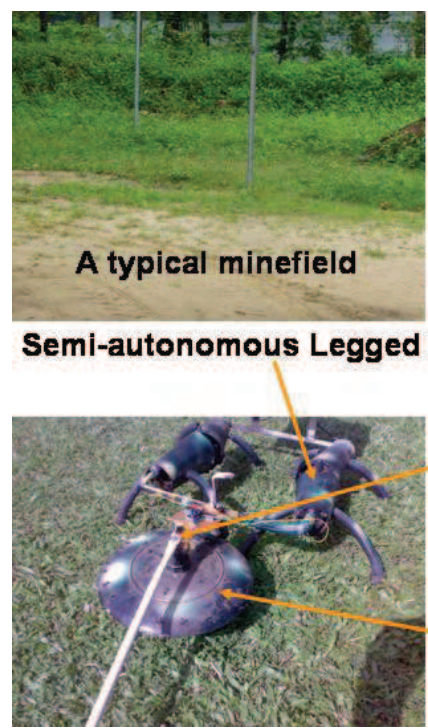

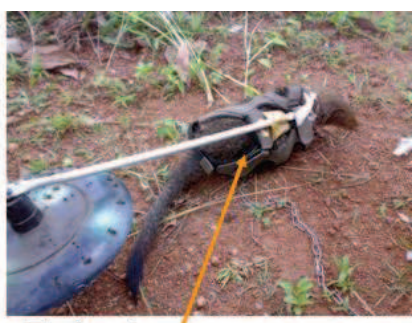

Trained mongoose attached to the robot

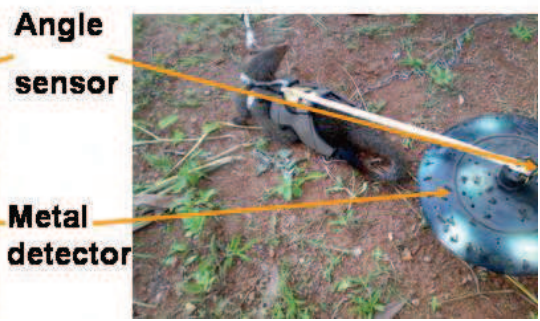

Fig. 2. How the mongoose is attached to the legged mobile robot called MURALI (Moratuwa University Robot for Anti-Landmine Intelligence)

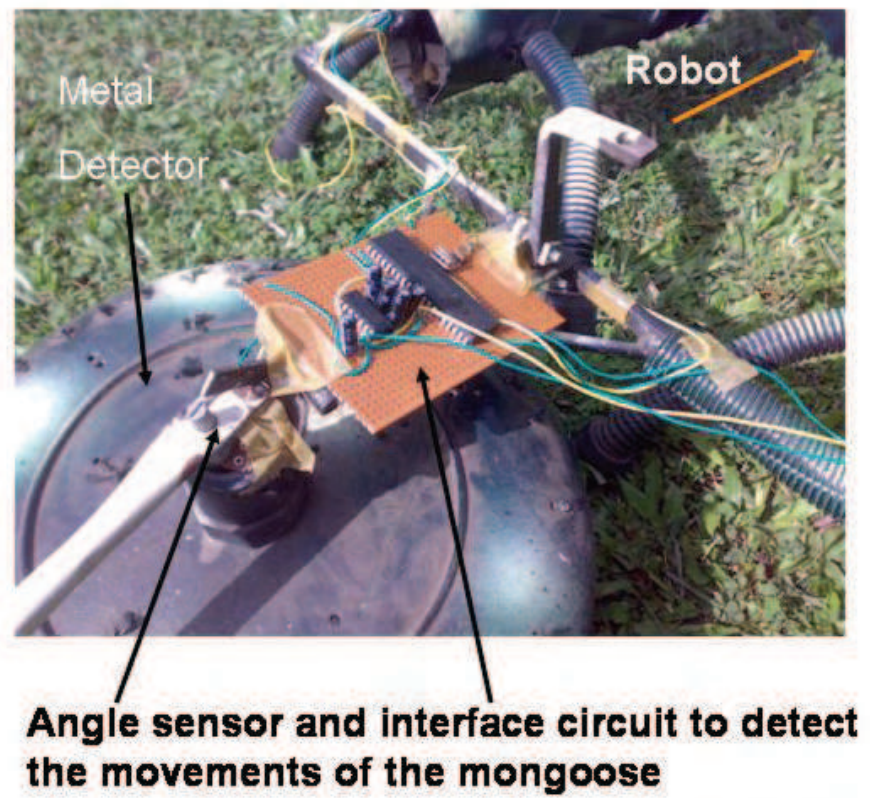

Fig. 3. How the angle sensor is interfaced to the slave PIC16f877 microcontroller 
Figure 3 shows how the robot's end of the cord that connects the robot to the mongoose is attached to an angle sensor. The sensor information was filtered and given to the analog port of a PIC16f877 slave microcontroller. The slave microcontroller is interfaced to a mother microcontroller that controls other sensors and actuators of the robot as shown in the schematic diagram in figure 4.

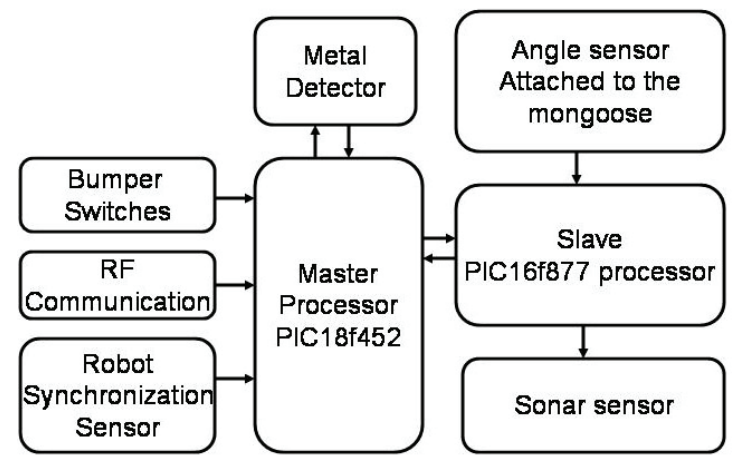

Fig. 4. The how different sensors and actuators are interfaced to the mother board of the robot

\subsection{Experimental Results of Robot-mongoose Integrated System}

Experiments were carried out by burying a small trace of explosives $<1 \mathrm{~g}$ just beneath the ground in different directions $\left(-45^{0},-30^{\circ},+30^{\circ}\right.$, and $\left.+45^{\circ}\right)$. The explosive trace was kept $3 \mathrm{~m}$ beyond the initial position of the mongoose. The robot sensed the direction of drag of the mongoose while the mongoose was sniffing for the explosives. Figure $\mathbf{5}$ shows that the mongoose takes less than 25 seconds to find the correct location after a brief phase of sniffing to trace the target.

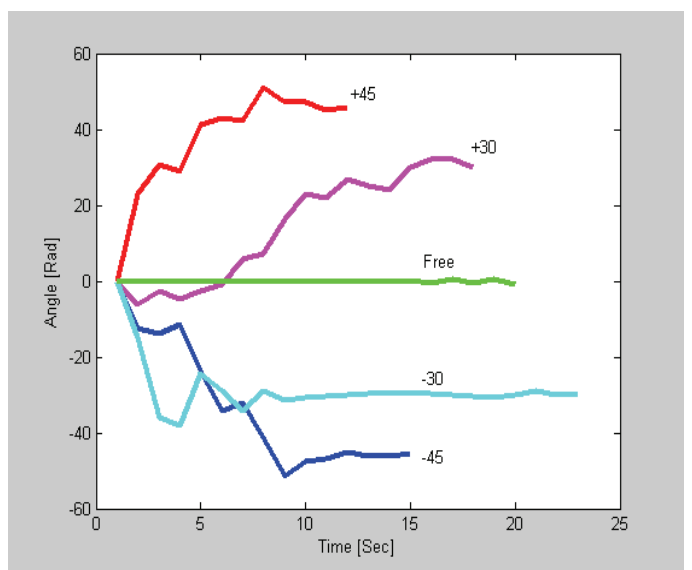

Fig. 5. The drag behavior of the mongoose for explosives buried in different target locations 


\subsection{Proposed Behavior Arbitration Mechanism in a System of a Rodent, Robot, and Human Systems that will Orchestrate Effective Navigation Behaviors}

The proposed system consists of a semi-autonomous robot, a trained animal to sniff for a target chemical, and a human trying to control the robot and understand the behaviors of the animal from a remote location as shown in figure 6 . Therefore, the final behavior of the system is an outcome of an interaction of three mutually dependant sub-systems with the surrounding environment.

Therefore, the effectiveness of the whole mission depends on how the behaviors recommended by each sub-system are arbitrated. Some of the published behavior arbitration mechanisms can be categorized into the following broad classes (Arkin R.C., 1998; Brooks R.A., 1986; Hoff J. \& Bekey G., 1995; Cooper R. \& Shallice T., 2000; Maes P, 1991).

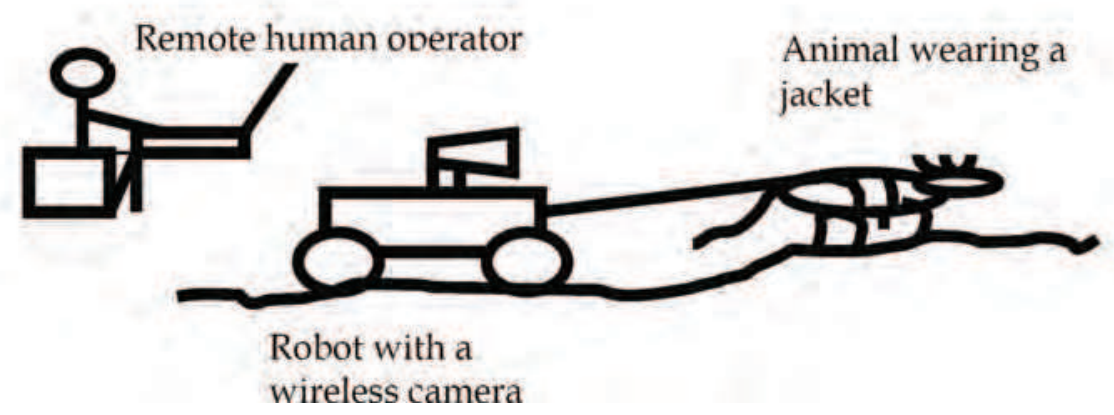

Fig. 6. The animal-robot-human system

\subsection{Voting for actions}

There is an arbitrator that votes for actions recommended by different behavior modules (Arkin R.C., 1998).

\subsection{Subsumption Architecture}

Behaviors are stacked in layers. The behaviors occupying higher layers can suppress those occupying lower layers given a situation [58].

\subsection{Vector Addition}

The actions recommended by each behavior are fused by taking the vector summation of the actions [59].

\subsection{Analysis of Each Sub-system in the Proposed Method}

In the proposed method, the human, robot, and the animal may influence the other subsystems in the following ways:

The human operator will watch visual feedback taken from the wireless camera mounted on the robot and send control commands through a wireless link to the robot. Therefore, he/she may influence the system in the following ways: 
1. Send control commands to keep the robot on track.

2. Command the robot to avoid obstacles.

3. Assist the animal to scrutinize an off track area by deviating the robot from the track.

4. Force the animal to avoid certain paths where the robot can not go, by controlling the robot to take an alternative path.

5. Control the speed of the robot relative to that of the animal to improve efficiency and to avoid intimidating the animal.

The semi-autonomous robot will execute its autonomous behaviors while accepting commands from the remote human controller. Therefore, it may influence the system in the following ways:

1. Keep the animal on a given track.

2. Use its autonomous behaviors to avoid obstacles.

3. Provide visual and haptic feedback to the human operator.

4. Reward the animal with a pleasing sound if it finds a target chemical.

5. The animal may influence the system in the following ways:

1. Drag the robot to different directions depending on the smell perception.

2. Force the robot to stop if it wants to scrutinize a location further.

3. Force the robot to accelerate and decelerate.

4. Elicit behaviors to alert the human.

5. Force the robot to avoid obstacles.

\subsection{Azimuth Control of the Robot}

At present the azimuth of controlled by the remote control commands sent by the human operator by watching the behaviors of the mongoose. The semi-autonomous behaviors of the robot contribute to intricate control actions in response to local conditions immediately in front of the robot. However, we propose an advanced behavior arbitration mechanism based on a weighted vector summation of actions recommended by each sub-system as given bellow.

Let, $\phi(k)$ is the azimuth of the robot, $\alpha(k)$ is the angle of drag of the animal, and $u(k)$ is the azimuth recommended by the human at time $k$. Therefore, the vectors $[\phi(k) \phi(k-1) \cdots \phi(k-N)],[\alpha(k) \alpha(k-1) \cdots \alpha(k-M)]$, and $[u(k) u(k-1) \cdots u(k-L)]$ represent the pattern of behavior of each sub-system in the recent past. The length of the vectors are decided by the order parameters given by $N, M$, and $L$.

Then, the azimuth of the robot at time $k+1$ is given by an adaptive autoregressive model given by $\phi(k+1)=\psi^{T} x$, where, $\psi^{T}=\left[a_{0} a_{1} \cdots a_{N-1} b_{0} b_{1} \cdots b_{M-1} c_{0} c_{1} \cdots c_{L-1}\right]$ is a vector of parameters and $x^{T}=[\phi(k) \phi(k-1) \cdots \phi(k-N) \alpha(k) \alpha(k-1) \cdots \alpha(k-M) u(k) u(k-1) \cdots u(k-L)]$.

The dynamics of behavior arbitration depends on the parameter vector $\psi$, and the order parameter vector $\left[\begin{array}{lll}N & M & L\end{array}\right]$. We envisage to study a method to adaptively change $[N M L]$ and $\psi$, so that the influence made by the behavior of each sub-system will adaptively change to orchestrate the best behavior. 


\subsection{Speed Control}

We tend to believe that the Subsumption architecture is suitable for speed control as shown in figure 6 . The robot and the animal negotiate a speed by vector addition. The human suppresses any decision taken by the system of the robot and the mongoose.

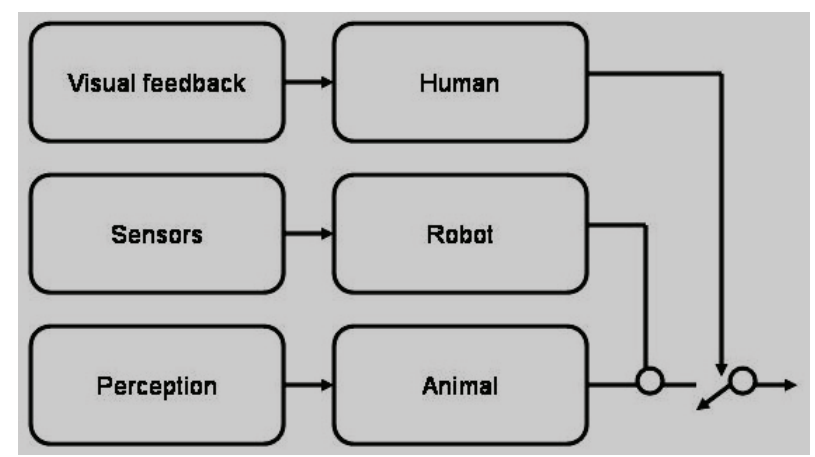

Fig. 6. The overall control algorithm of the robot

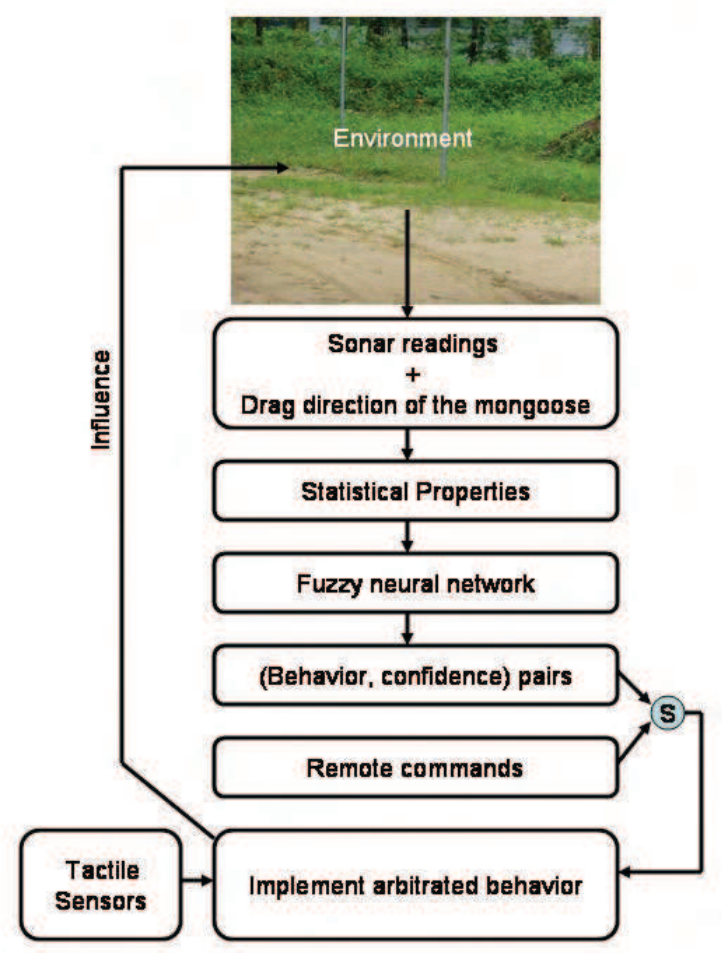

Figure 6 shows the proposed control algorithm of the human-robot-animal integrated system to detect landmines. 


\section{Discussion}

To the best of the author's knowledge, this is the first time a human-robot-animal integrated system is tested for antipersonnel landmine detection. The proposed system tries to integrate distinct capabilities of three different systems to improve the effectiveness of landmine detection in a cluttered environment. The mongoose is found to be a rodent with extremely sensitive olfactory capabilities, dexterous navigation capabilities in a cluttered environment, and small enough to burrow through rubble. The lightweight legged robot $(4 \mathrm{~kg})$ can move in a minefield without detonating landmines, carry a metal detector, and interact with the mongoose and the human. The remote human operator can analyze the behaviors of the animal-robot system and judge how best the system should move from a remote location. Therefore, the system achieves a fundamental objective of humanitarian landmine detection by improving the effectiveness and accelerating the detection process through removing the human operator from the minefield. The design gave much emphasis on reducing the need to have expensive sensors and sophisticated image processing systems in order to make it as cost effective and reliable as possible. Therefore, there were only a single sonar proximity sensor and two bumper switches attached to the front of the robot. However, further improvements are needed in the arbitration mechanism that optimizes the synergy among the human, robot, and the animal by improving the learning algorithms. The robot can learn from both the animal and the human though the teaching signals can be noisy. The animal can learn from both the human and the robot to navigate with the robot attached to it. The human can learn from the animal and the robot by observing the limitations of the animal-robot system. We are conducting further research on learning algorithms that suits this scenario. Commensurate efforts have to be taken to simplify the learning algorithms to suit commercially available embedded processors and to improve the processor network to accommodate the extra processing load. Furthermore we hope to automate the training process of mongooses based on the wealth of knowledge we have gathered through manual training. This will allow the trainers to run the training sessions round the clock.

\section{Acknowledgements}

This work was supported by the Sri Lanka National Science Foundation Grant: G/2006/TDPS/02. The authors extend special thanks to Brigadier Chrishantha de Silva, Brigade Commander of the Sri Lanka Engineer's Brigade, for providing valuable technical advices, staff and infrastructure to carry out the experiments, Captain Thushara Jayawardana and Corporal Pradeep Kumara for coordinating the logistics and assisting to carry out experiments.

\section{References}

Arkin R.C. (1998.), “Behavior-Based Robotics” MIT Press, Cambridge, MA.

Ayache N. and Faugeras O.D. (1989.), "Maintaining Representation of the Environment of a Mobile Robot", IEEE Trans. on Robotics and Automation, pp. 804 - 819, vol. 5, no. 6.

Bohlen M. (1999.), “A Robot in a Cage”, proc. of IEEE International Symposium on

Computational Intelligence in Robotics and Automation, pp. 214 - 219. 
Bozma O. and Kuc R. (1991.), “Building a Sonar Map in a Specular Environment using a Single Mobile Sensor", IEEE Trans. on Pattern Analysis and Machine Intelligence, pp. 12601269, vol. 13, no. 12.

Brashers-Krug T., Shadmehr R., and Bizzi E. (1996), "Consolidation in human motor memory", Nature, pp. 252-255, vol. 382.

Brooks R.A. (March 1986.), "A Robust Layered Control System for a Mobile Robot", IEEE Journal of Robotics and Automation, pp. 14 - 23, vol. RA-2, no.1.

Bunyan M. and Barratt J. (4th of February, 2002), "AMS Guidance on Technology readiness Levels (TRLs)", GBG/36/10, UK MOD.

Burgard W., Fox D., Hennig D., and Schimidt T. (1998.), "Estimating the Absolute Position of a Mobile Robot using Position Probability Grids", In Procs. of the Thirteen national Conference on Artificial Intelligence, Menlo Park, AAAI Press/MIT Press.

Caprari G., Colot A., Seigwart R., Halloy J., and Deneubourg J.L. (June 2005.), “Animal and Robot Mixed Societies-Building Cooperation Between Micro robots and Cockroaches", IEEE Robotics and Automation Magazine, pp. 58 - 65.

Chong K.S. and Kleeman L. (1996.), "Mobile Robot Map Building from an Advanced Sonar Array and Accurate Odometry", Technical Report MECSE-1996-10, Department of Electrical and Computer Systems Engineering, Monash University, Melbourne.

Collins L., Gao P., Schofield D., Moulton J.P., Makowsky L.C., Deidy D.M., and Weaver R.C. (April 2002.), “A statistical approach to landmine detection using broadband electromagnetic induction data", IEEE trans. on geoscience and remote sensing, vol. 40, no. 4 .

Cooper R. and Shallice T. (2000.), "Contention Scheduling and the Control of Routine Activities", Cognitive Neurophysiology, pp. 298 - 338, vol. 17, no. 4.

Crabbe S., Eng L., Gårdhagen P., Berg A. (2005) “Detection of explosive as an indicator of landmines - BIOSENS project methodology and field tests in South East Europe ", Proceedings of SPIE Conference on Detection and Remediation Technologies for Mines and Mine-like Targets X, Vol. 5794, pp. 762 - 773, Orlando.

BIOSENS consortium (2004) BIOSENS Final Report, www.eudem.info

Crabbe S., Sachs J., Alli G., Peyerl P., Eng L., Khalili M., Busto J. and Berg A. (2004) “Results of field testing with the multi-sensor DEMAND and BIOSENS technology in Croatia and Bosnia developed in the European Union's 5th Framework Program", Proceedings of SPIE Conference on Detection and Remediation Technologies for Mines and Minelike Targets IX, Vol. 5415.

Cumming C., Aker C., Fisher M., Fox M., laGrone M., Reust D., Rockley M., Swager T., Towers E. and Williams V. (2001), "Using Novel Fluorescent Polymers as Sensory Materials for Above-Ground Sensing of Chemical Signature Compounds Emanating from Buried Landmines." IEEE Transactions on Geoscience and Remote Sensing, Vol. 39, No. 6, pp. 1119-1128.

Daniels D.J., Curtis P., Amin R., Hunt N. (2005), "MINEHOUND Production Development", In Procs. of SPIE Conference on Detection and Remediation Technologies for Mines and Mine-like Targets X, pp. 488 - 494, Vol. 5794, Orlando, US.

Dayan P., Balleine B. (2002), "Reward, Motivation, and Reinforcement Learning", Neuron, pp. 285-298, vol. 36, Issue 2.

De Schutter G., Theraulaz G., and Deneubourg J.L. (2001), “Animal-Robot Collective 
Intelligence", Annals of Mathematics and Artificial Intelligence, pp. 223 - 238, vol. 31.

Doheny R.C., Burke S., Cresci R., Ngan P., Walls R. (2005.), “Handheld Standoff Mine Detection System (HSTAMIDS) Field Evaluation in Thailand", In Procs. of SPIE Conference on Detection and Remediation Technologies for Mines and Mine-like Targets X, pp. 889-900, Vol. 5794, Orlando, US.

Donskoy D.M. (October, 1998) Non-linear seismo-acoustic technique for landmine detection And discrimination, Second International Conference on Detection of Abandoned Land Mines, IEE Conference Publication No. 458, pp. 244-248, Edinburgh, UK.

Dudek G., Freedman R., and Rekleitis I.M. (1996.), “Just-in-time sensing: Efficiently Combining Sonar and Laser Range Data for Exploring Unknown Worlds", Proc. of the IEEE International Conference on Robotics and Automation, pp. 667-672, Washington.

Ferrari S. and Vaghi A. (April 2006.), "Demining sensor Modeling and feature-level fusion by Bayesian networks", pp. 471 - 483, IEEE Sensors Journal, vol. 6, no. 2.

Fisher M. E., Sikes J. (2003.) "Minefield edge detection using a novel chemical vapor sensing technique", Proceedings of SPIE Conference on Detection and Remediation Technologies for Mines and Mine-like Targets VIII, Vol. 5089, pp. 1078-1087, Orlando, US.

Forsman P. and Halme A. (June 2005.), “3-D Mapping of Natural Environments with Trees by Means of Mobile perception", IEEE Trans. on Robotics, pp. 482 - 490, vol. 21, no. 3.

George V., Jenkins T.F., Leggett D.C., Cragin J.H., Phelan J., Oxley J. and Pennington J. (1999), "Progress on Determining the Vapor Signature of a Buried Landmine." Proc. SPIE, Detection and Remediation Technologies for Mines and Minelike Targets IV, vol. 3710, part 2, p. 258-269.

German P.W. and Fields H.L. (March, 2007.), "Rat Nucleus Accumbens Neurons Persistently Encode Locations Associated With Morphine Reward", Journal of Neurophysiology, pp. 2094 - 2106, vol. 97, no. 3.

Girard M. (June 1987.), "Interactive Design of 3D Computer-Animated legged Animal Motion”, Proc. of the IEEE, pp. 39 - 51.

Goldberg G. and Matari'c M. (1997.), "Interference as a Tool for Designing and Evaluating Multirobot Controllers", In Procs of the 14th National Conference on Artificial Intelligence (AAAI-97), pp. 637-642, MIT Press, Cambridge, MA, USA.

Gonzalez J. (1992.), “An Iconic Position Estimator for a 2D Laser Rangefinder”, Proc. of the IEEE International Conference on Robotics and Automation, pp. 2646-2651, Los Alamitos, CA:

Hayes A. (2002.) "How many Robots? Group Size and Efficiency in Collective Search Tasks", In Procs. of the 6th International Symposium on Distributed Autonomous Robotic Systems (DARS-02), H. Asama, T. Arai, T. Fukuda, and T. Hasegawa, Eds. Springer Verlag, Heidelberg, Germany, pp. 289-298.

Hoff J. and Bekey G. (Nov/Dec, 1995), "architecture for behavior coordination learning", in proc. of IEEE International Conference on Neural Networks, pp. 2375 - 2380, vol.5, Issue.

Hollerman J.R., Tremblay L., and Schultz W. (August 1998.), “Influence of Reward Expectation on Behavior-Related Neuronal Activity in Primate Striatum", Journal of Neurophysiology, pp. 947 - 963, vol. 80, no. 2.

Holzer R. and Shimoyama I. (1997.), “Locomotion Control of a Bio-Robotic System via Electric 
Stimulation", in Proc. of IEEE/RSJ IROS International Conference on Intelligent Robots and Systems, pp. 1514 - 1519, vol. 3, Grenoble, France.

Ishii H., Nakasuji M., Motonori Ogura, Miwa H., and Takanashi A. (April 2005.)

,"Experimental Study on Automatic Learning Speed Acceleration for a Rat using a Robot", Prod. Of the IEEE International Conference on Robotics and Automation, pp. 3078 - 3083, Barcelona, Spain.

Jones C. and Matari'c M. (2003.), "Adaptive Division of Labor in Large-Scale Minimalist MultiRobot Systems", In Procs. of IEEE/RSJ International Conference on Intelligent Robots and Systems, pp. 1969-1974, vol. 2, IEEE Press, New York, NY, USA.

Krieger M. and Billeter J.B. (2000), "The Call of Duty: Self-Organized Task Allocation in a Population of up to Twelve Mobile Robots", Robotics and Autonomous Systems, pp. 65-84, vol. 30, no. 1-2.

Maes P. (1991.), "A Bottom-Up Mechanism for Behavior Selection in an Artificial Creature” In From Animals to Animates, MIT Press.

Mataric M.J. (1994.), "Reward functions for accelerated learning", In Procs of the Eleventh International Conference on Machine Learning, pp. 181-189, Morgan Kaufmann, San Francisco, CA.

Mataric M.J. (2000.), "Sensorymotor primitives as a basis for imitation: linking perception to action and biology to robotics", In C. Nehaniv and K. Dautenhahn, editors, Imitation in Animals and Artifacts. The MIT Press, Cambridge, MA.

Montague P. and Berns G. (2002), "Neural Economics and the Biological Substrates of Valuation", Neuron, pp. 265-284, vol. 36, Issue 2.

Ng A.Y., Harada D., and Russell S. (1999.), "Policy invariance under reward transformations: Theory and applications to reward shaping", In Procs of the Sixteenth International Conference on Machine Learning, pp. 278-287, Morgan Kaufmann, San Francisco, CA.

Perrin S., Duflos E., Vanheeghe P., and Bibaut A. (November 2004.), "Multisensor fusion in the frame of evidence theory for landmine detection", IEEE trans. on systems, man, and cybernetics-Part C: Applications and reviews, pp. 485 - 498, vol. 34, no. 4.

Ramanan D., Forsyth D.A., and Bernard K. (August 2006.), "Building models of animals from video", IEEE trans. on pattern analysis and machine intelligence, pp. 1319 - 1334, vol.28, no. 8 .

Sato M, Fujiwara J., Feng X., Kobayashi T. (September 2005) “Dual Sensor ALIS evaluation in Afghanistan", IEEE Geoscience and Remote Sensing Society Newsletter, pp. 22-27.

Savelyev T.G., Kempen L.V., Sahli H., Sachs J., and Sato M. (January, 2007.), “Investigation of time-frequency features for GPR landmine discrimination", IEEE trans. On Geoscience and Remote Sensing, pp. 118 - 129, vol. 45, no. 1.

Schultz W., Dayan P., and Montague P.R. (1997), "A Neural Substrate of Prediction and Reward", Science, pages 1593-1598, ISSUE 5306.

Scott W.R., martin J.S., and Larson D. (June 2001.), “Experimental model for a seismic landmine detection system", IEEE trans. on geosciences and remote sensing, pp. 1155 - 1164, vol. 39 , no. 6 ,

Shadmehr S. and Holcomb H.H. (1997.), "Neural correlates of motor memory consolidation". Science, pp. 821-825, vol. 277.

Sun Y.and Li J. (August 2003.), “Time-frequency analysis for plastic landmine detection via 
Forward-looking ground penetrating radar", IEE Proc. on radar Sonar Navigation, vol. 150, no. 4, pp, 253.

Sutton R.S. and Barto A.G. (1998.), "Reinforcement Learning", MIT Press.

Talwar S.K., Xu S., Hawley E.S., Weiss S.A., Moxon K.A., Chapin J.K. (May 2002.), “Rat Navigation Guided by Remote Control", Nature, pp. 37 - 38, vol. 417.

Tan Y., Tantum S.L., and Collins L.M. (July 2005.), "Kalman filtering for enhanced landmine detection using Quadrupole resonance", IEEE trans. On Geoscience and Remote Sensing, pp. 1507 - 1516, vol. 43, no. 7.

Thoroughman K. and Shadmehr R. (2000.), "Learning of action through adaptive combination of motor primitives", Nature, pp. 742-747, vol. 407.

Thrishantha Nanayakkara, Lasitha Piyathilaka, and Akila Subasingha, "A Simplified Statistical Approach to Classify Vegetated Environments for Robotic Navigation in Tropical Minefields", In Procs. Of the Internatonal Conference on Information and Automation, pp. 337 - 342, December 15 - 18, Colombo, Sri Lanka.

Thrun S., Burgard W., and Fox D. (July 1998.), “A Probabilistic Approach to Concurrent Mapping and Localization for Mobile Robots", Journal of Autonomous Robots, pp. 253 - 271, vol. 5, no. 3-4.

Vaska P., Woody C.L., Schlyer D.J., Shokouhi S., Stoll S.P., Connor P.O., Junnarkar S.S., Rescia S., Yu B., PurschkeM., Kandasamy A., Villanueva A., Kriplani A., Volkow A., Lecomte R., and Fontaine R. (October 2004.), "RatCAP: Miniaturized HeadMounted PET for conscious Rodent Brain Imaging", IEEE trans. on Nuclear Science, vol. 51, No. 5.

Watkins. C. J. C. H. (1989.), “Learning from Delayed Rewards”, Ph.D. Dissertation, Cambridge University.

Xiang N. and Sabatier J. (October 2004.), “Laser Doppler vibrometer-based acoustic landmine detection using the fast M-sequence transform", IEEE trans. on geosciences and remote sensing letters, pp. 292 - 294, vol.1, no. 4.

Xu X., Miller E.L., Rappaport C.M., and Sower G.D. (April 2002.),"Statistical method to detect subsurface objects using array ground-penetrating radar data", IEEE trans. on geoscience and remote sensing, pp. 963 - 976, vol. 40, no. 4.

Ziegler S.I. (April 2005.), "Development of a Small-Animal PET System", Proc. of the IEEE, pp. 763 - 770, vol.93, no. 4. 


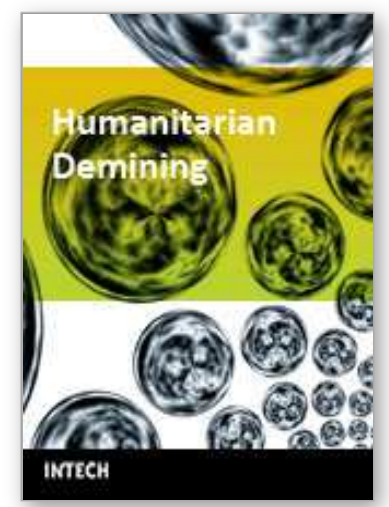

\author{
Humanitarian Demining \\ Edited by Maki K. Habib
}

ISBN 978-3-902613-11-0

Hard cover, 392 pages

Publisher I-Tech Education and Publishing

Published online 01, February, 2008

Published in print edition February, 2008

United Nation Department of Human Affairs (UNDHA) assesses that there are more than 100 million mines that are scattered across the world and pose significant hazards in more than 68 countries. The international Committee of the Red Cross (ICRC) estimates that the casualty rate from landmines currently exceeds 26,000 persons every year. It is estimated that more than 800 persons are killed and 1,200 maimed each month by landmines around the world. Humanitarian demining demands that all the landmines (especially AP mines) and ERW affecting the places where ordinary people live must be cleared, and their safety in areas that have been cleared must be guaranteed. Innovative solutions and technologies are required and hence this book is coming out to address and deal with the problems, difficulties, priorities, development of sensing and demining technologies and the technological and research challenges. This book reports on the state of the art research and development findings and results. The content of the book has been structured into three technical research sections with total of 16 chapters written by well recognized researchers in the field worldwide. The main topics of these three technical research sections are: Humanitarian Demining: the Technology and the Research Challenges (Chapters 1 and 2), Sensors and Detection Techniques for Humanitarian Demining (Chapters 3 to 8 ), and Robotics and Flexible Mechanisms for Humanitarian Demining respectively (Chapters 9 to 16$)$.

\title{
How to reference
}

In order to correctly reference this scholarly work, feel free to copy and paste the following:

Thrishantha Nanayakkara, Tharindu Dissanayake, Prasanna Mahipala and K. A. Gayan Sanjaya (2008). A Human-Animal-Robot Cooperative System for Anti-Personal Mine Detection, Humanitarian Demining, Maki K. Habib (Ed.), ISBN: 978-3-902613-11-0, InTech, Available from:

http://www.intechopen.com/books/humanitarian_demining/a_human-animal-

robot_cooperative_system_for_anti-personal_mine_detection

\section{INTECH}

open science | open minds

\section{InTech Europe}

University Campus STeP Ri

Slavka Krautzeka 83/A

51000 Rijeka, Croatia

Phone: +385 (51) 770447

\section{InTech China}

Unit 405, Office Block, Hotel Equatorial Shanghai

No.65, Yan An Road (West), Shanghai, 200040, China

中国上海市延安西路65号上海国际贵都大饭店办公楼 405 单元

Phone: +86-21-62489820 
Fax: +385 (51) 686166

Fax: +86-21-62489821

www.intechopen.com 
(C) 2008 The Author(s). Licensee IntechOpen. This chapter is distributed under the terms of the Creative Commons Attribution-NonCommercialShareAlike-3.0 License, which permits use, distribution and reproduction for non-commercial purposes, provided the original is properly cited and derivative works building on this content are distributed under the same license. 